\section{COMPORTAMIENTO POSTCOSECHA DE FRUTOS DE ILAMA (Annona diversifolia) EN MADUREZ COMESTIBLE ALMACENADOS EN ATMÓSFERA MODIFICADA}

\section{POSTHARVEST BEHAVIOR OF ILAMA (Annona diversifolia) FRUITS AT EDIBLE MATURITY STORED IN A MODIFIED ATMOSPHERE}

\author{
Salvador Valle-Guadarrama ${ }^{1 \star}$, Xóchitl G. \\ Ruiz-Sánchez ${ }^{1}$, Crescenciano Saucedo-Veloz ${ }^{2}$, Adalberto \\ Gómez-Cruz ${ }^{1}$ y Lila M. Marroquín-Andrade ${ }^{3}$
}

${ }^{1}$ Departamento de Ingeniería Agroindustrial y ${ }^{3}$ Departamento de Fitotecnia, Universidad Autónoma Chapingo (UACh). Carr. México-Texcoco $\mathrm{km}$ 38.5, 56230, Chapingo, Edo. de México, México. ${ }^{2}$ Fruticultura, Colegio de Postgraduados, Carr. México-Texcoco km 36.5, 56230, México, México.

*Autor para correspondencia (svalleg@taurus.chapingo.mx)

\section{RESUMEN}

El fruto de ilama o papausa (Annona diversifolia Saff.) tiene potencial alto de comercialización, pero esto se limita porque su vida de anaquel es muy corta, porque los productores lo cosechan cuando llega a madurez comestible, y porque se desconoce su respuesta al uso de tecnologías para aumentar la vida útil. El objetivo del trabajo fue evaluar el efecto del uso de atmósferas modificadas (AM) en la calidad postcosecha de frutos de ilama en madurez de consumo almacenados a $20^{\circ} \mathrm{C}$. Se realizaron almacenamientos durante $9 \mathrm{~d}$ en atmósfera de aire natural (AN) y en AM con polietileno de baja densidad de $33 \mu \mathrm{m}$ de espesor. Los frutos de AM tuvieron $91 \%$ menos pérdida de peso y mayores valores de firmeza $(1.2 \mathrm{~N})$, en comparación con los de AN. La respiración fue de $97.1 \mathrm{~mL} \mathrm{~kg} \mathrm{~h}^{-1}$, sin diferencia entre tratamientos. Tampoco hubo diferencia en contenido de acidez, sólidos solubles totales y azúcares totales, cuyos valores promedio fueron de $0.20 \%, 17.9$ ${ }^{\circ}$ Brix y $\mathbf{1 7 . 6} \%$, respectivamente. Sin embargo, el contenido de azúcares reductores, que varió entre 4 y $10 \%$, fue mayor en el material manejado en AM. La atmósfera modificada probó ser útil para reducir las velocidades de pérdida de peso y ablandamiento, lo que puede permitir un tiempo de manejo postcosecha mayor de los frutos de ilama.

Palabras clave: Annona diversifolia, papausa, atmósferas modificadas, calidad postcosecha.

\section{SUMMARY}

The ilama fruit or papausa (Annona diversifolia Saff.) has high commercialization potential, but this is limited due to its high perishability, edible maturity at harvesting, and its responses to technologies focused to extend shelf life are unknown. The objective of the work was to evaluate the effect of using modified atmospheres (MA) on the postharvest quality of ilama fruits at ripe maturity stored at $20^{\circ} \mathrm{C}$. Two treatments were evaluated during a period of $9 \mathrm{~d}$ : storage in MA with low density polyethylene of $33 \mathrm{~mm}$ thickness, and storage in natural air (NA). Fruits of MA had $91 \%$ less weight loss and higher firmness values (1.2 N) than those of NA. The respiratory activity had an average value of $97.1 \mathrm{~mL} \mathrm{~kg}^{-1} \mathrm{~h}^{-1}$, without difference between treatments. There was not difference either in contents of acidity, total soluble solids and total su- gars, whose average values were $0.20 \%, 17.9 \circ$ Brix and $17.6 \%$, respectively. However, reducing sugars content, which varied between 4 and 10 $\%$, had the highest values in material handled in MA. The modified atmosphere proved to be useful for reducing weight losses and softening rate of ilama fruits, thus allowing longer postharvest storage periods.

Index words: Annona diversifolia, papausa, modified atmospheres, postharvest quality.

\section{INTRODUCCIÓN}

La familia de las Anonáceas comprende alrededor de 50 géneros, entre los cuales Annona, Rollinia y Asimina producen frutos comestibles y los dos primeros son de importancia comercial (George y Nissen, 2003). La mayoría de las especies del género Annona provienen de Suramérica y América Central (Pinto, 2005); México se reconoce como centro de origen de Annona longifolia (Jalisco) y A. longipes (Veracruz), en tanto que la región del Sureste de México y Guatemala es el origen de A. diversifolia, A. purpurea y A. scleroderma (Ferreira y Pinto, 2005).

Las anonas son consumidas como frutos frescos, productos semi-procesados y procesados, especialmente en postres (Cordeiro et al., 2005); además poseen aplicaciones medicinales (González-Trujano et al., 2009) y nutracéuticas (Julián-Loaeza et al., 2011). Según Pareek et al. (2011), sus frutos se clasifican como climatéricos, con respiración que puede tener comportamiento mono o bifásico y ascender hasta $350 \mathrm{~mL} \mathrm{CO} \mathrm{kg}^{-1} \mathrm{~h}^{-1}$ a $25-30^{\circ} \mathrm{C}$ en algunas especies, con producción de etileno que puede alcanzar valores entre 46.2 y $68.5 \mu \mathrm{L} \mathrm{kg}^{-1} \mathrm{~h}^{-1}$ en algunos cultivares de chirimoya (A. cherimola Mill.), lo cual se traduce en un ablandamiento rápido de la pulpa que dificulta el manejo durante el transporte y la comercialización. Asimismo, estos autores señalaron que estos frutos pueden experimentar daño por frío y la temperatura mínima que resisten varía entre 7 y 15 ${ }^{\circ} \mathrm{C}$ en función de la especie.

El fruto de ilama o papausa (A. diversifolia Saff.) desarrolla peso entre 500 y $900 \mathrm{~g}$. La pulpa, que puede alcanzar hasta $50 \%$ del fruto, ostenta sabor y consistencia agradables al paladar (Julián-Loaeza et al., 2011; Moreno-Velázquez et al., 2008). Según Julián-Loaeza et al. (2011), este fruto posee capacidad antioxidante $\left(\mathrm{IC}_{50}\right.$ ) que varía de 1700 a 2000 $\mu \mathrm{g} \mathrm{mL}^{-1}$, situación que le confiere potencial de comercialización en estado fresco y procesado. Saucedo-Veloz y Arévalo-Galarza (2004) señalaron que la mayor demanda del fruto es en fresco, y que factores como estado de madurez al momento de cosecha, incidencia de pudriciones y elevado metabolismo, limitan significativamente su vida de anaquel en condiciones ambientales.

A este respecto, el criterio de cosecha de los productores regionales se identifica con el inicio de agrietamiento de la cáscara en la zona peripeduncular, como parte del fenómeno natural de dehiscencia, y se considera el índice 